\title{
Guidelines for the genetic diagnosis of hereditary recurrent fevers
}

\author{
Y Shinar, ${ }^{1}$ L Obici, ${ }^{2}$ I Aksentijevich, ${ }^{3}$ B Bennetts, ${ }^{4}$ F Austrup, ${ }^{5}$ I Ceccherini, ${ }^{6}$ \\ J M Costa, ${ }^{7}$ A De Leener, ${ }^{8}$ M Gattorno, ${ }^{9}$ U Kania, ${ }^{10}$ I Kone-Paut, ${ }^{11}$ S Lezer, ${ }^{12}$ \\ A Livneh, ${ }^{13}$ I Moix, ${ }^{14}$ R Nishikomori, ${ }^{15}$ S Ozen, ${ }^{16}$ L Phylactou $^{17}{ }^{17}$ Risom, ${ }^{18}$ \\ D Rowczenio, ${ }^{19}$ T Sarkisian, ${ }^{20}$ M E van Gijn, ${ }^{21}$ M Witsch-Baumgartner, ${ }^{22}$ M Morris, ${ }^{23}$ \\ H M Hoffman, ${ }^{24}$ I Touitou ${ }^{25}$
}

- Additional material is published online only. To view these files please visit the journal online (http://dx.doi.org/10.1136/ annrheumdis-2011-201271).

For numbered affiliations see end of article

\section{Correspondence to} Isabelle Touitou, Unité médicale des maladies autoinflammatoires, CHRU

Montpellier, INSERM

U844, Université UM1

Montpellier, France:

isabelle.touitou@inserm.fr

Accepted 8 April 2012

\section{ABSTRACT}

Hereditary recurrent fevers (HRFs) are a group of monogenic autoinflammatory diseases characterised by recurrent bouts of fever and serosal inflammation that are caused by pathogenic variants in genes important for the regulation of innate immunity. Discovery of the molecular defects responsible for these diseases has initiated genetic diagnostics in many countries around the world, including the Middle East, Europe, USA, Japan and Australia. However, diverse testing methods and reporting practices are employed and there is a clear need for consensus guidelines for HRF genetic testing.

Draft guidelines were prepared based on current practice deduced from previous HRF external quality assurance schemes and data from the literature. The draft document was disseminated through the European Molecular Genetics Quality Network for broader consultation and amendment. A workshop was held in Bruges (Belgium) on 18 and 19 September 2011 to ratify the draft and obtain a final consensus document. An agreed set of best practice guidelines was proposed for genetic diagnostic testing of HRFs, for reporting the genetic results and for defining their clinical significance.

\section{INTRODUCTION}

Patients with hereditary recurrent fevers (HRFs) present with recurrent bouts of fever and inflammatory symptoms involving, in particular, the abdomen, joints and skin. ${ }^{12}$ The causative genes for HRFs encode proteins involved in the regulation of innate immunity, mainly by affecting proinflammatory cytokines and apoptosis pathways. While familial Mediterranean fever (FMF) is relatively common in several Mediterranean and Middle Eastern populations, ${ }^{3}$ most HRFs are rare diseases. The best-characterised HRFs are two recessively inherited diseases: (FMF, gene MEFV, MIM 608107) and mevalonate kinase deficiency ( $\mathrm{MKD}$, gene $M V K$, MIM 251170) and two dominantly inherited diseases: tumour necrosis factor (TNF) receptor-associated periodic syndrome (TRAPS, gene TNFRSF1A, MIM 191190) and cryopyrin-associated periodic syndrome (CAPS, gene NLRP3, MIM 606416). Patients with HRF often display similar inflammatory symptoms with variable intensity and localisation of symptoms, making their clinical diagnosis difficult.
Since the discovery of these four HRF genes, almost 700 nucleotide variants have been identified and recorded in Infevers, a database dedicated to autoinflammatory sequence variants (http:// fmf.igh.cnrs.fr/ISSAID/infevers/). ${ }^{4-6}$ Some of these variants are clearly pathogenic, but most are unconfirmed or seemingly non-pathogenic variants. A significant number of patients clinically diagnosed with recessive HRFs have been found to carry only one disease-associated mutation in the respective genes $^{7}$ despite extensive searching for a second pathogenic mutation in the coding region, ${ }^{89}$ and continuing search for mutations affecting regulatory sequences or transcript splicing that would affect gene expression. ${ }^{10} 11$

Genetic testing for HRF is a logical and feasible way to corroborate clinical diagnosis. ${ }^{12} 13$ Five-year experience of external quality assessment and proficiency testing (PT) (external quality assurance/PT) conducted between 2006 and 2010 showed that although there has been an impressive improvement in the quality of HRF testing and reporting, many issues still remain to be addressed and standardised. ${ }^{14}$ Guidelines using the standard definition by Field and Lohr ${ }^{15}$ are now proposed to provide a framework for best laboratory practice and reporting on the genetic diagnosis of HRFs as agreed by an international consortium of experts in the field. They are intended to be used primarily by molecular geneticists and by other health professionals involved in the care of these patients.

\section{METHODOLOGY}

A draft report was written by the organisers and assessors of the European Molecular Genetics Quality Network for HRFs with reference to relevant literature, reviews of reports issued during the five previous international HRF meetings, web-based resources relating to the subject and examples of guidelines for other hereditary diseases (eg, haemochromatosis, ${ }^{16}$ cystic fibrosis ${ }^{17}$ and von Willebrand ${ }^{18}$ diseases). The draft was disseminated to molecular geneticists and clinicians working in the field of HRFs and discussed with them during a best practice workshop held in Bruges (Belgium) on 18 and 19 September 2011. In the light of feedback of the participants, amendments were made and a second draft was disseminated by email, after which, the final document was ratified. 


\section{INDICATIONS FOR HRF TESTING}

\section{Minimal requirements for the genetic test}

We suggest that the following minimum set of requirements should be obtained: patient name, date of birth, gender, ethnicity/origin, written informed consent (depending on countryspecific law), referring doctor's name and contact details of the person who will receive the results.

\section{Symptomatic patients}

The main indication for genetic testing of HRFs is in the case of a patient with a clinical symptom pattern consistent with one or more of the syndromes. Thus, clinical data that justify the choice of one or more HRFs genetic tests are required. It is not unusual that when overlapping, partial or atypical clinical symptoms impede an accurate clinical diagnosis, screening of several HRF-responsible genes gives the correct diagnosis.

The clinical HRF referral usually includes the frequency of attacks, duration, sites affected, acute phase reactants levels, biomarkers for mevalonic aciduria or amyloidosis and a letter by an expert clinician. An example of a clinical chart is provided in online supplementary figure S1. It has been established in France by GenMAI, the national network for genetic diagnosis of autoinflammatory diseases, in conjunction with the clinical reference centres. Decision trees for genetic diagnosis in atypical patients and patients with sporadic disease have been proposed. ${ }^{13}{ }^{19}$ In addition, a diagnostic score for children with periodic fever has been elaborated in Italy. ${ }^{20}$

\section{Presymptomatic diagnosis and carrier status}

In general, presymptomatic diagnosis is not advisable, as its interpretation is inconclusive, may be complicated for mutations with incomplete penetrance and it usually does not call for medical intervention. Presymptomatic testing may be recommended after genetic counselling for asymptomatic family members when a severe genotype has been found in relatives with an overt disease, or if there is a family history of amyloidosis. Follow-up of people at risk may avoid occurrence of this life-threatening complication. However, whether such cases should be given prophylactic treatment remains controversial. Evaluation of carrier status could be recommended in healthy relatives to phase two known disease-associated or new mutations, when identified in an affected patient.

\section{Prenatal diagnosis and preimplantation genetic diagnosis}

Generally prenatal diagnosis (PND) and preimplantation genetic diagnosis (PGD) are not considered appropriate for HRF as most of these conditions are treatable and symptoms usually decrease over time. However, it may be appropriate to discuss PND or PGD in families affected by a particularly severe form of $\mathrm{MKD}$ or chronic infantile neurological, cutaneous and articular syndrome (CINCA) as these disorders can be associated with debilitating complications: blindness, deafness, mental retardation, ataxia and bone deformation. However, most severe CINCA-associated mutations occur de novo and this should be considered before offering PND and PGD. If PND is planned, it should be performed after genetic counselling.

\section{DIAGNOSTIC STRATEGY}

\section{Diagnostic laboratories and expert structures}

Genetic diagnosis for HRFs is now widely available. There are 99 laboratories providing FMF testing in Europe (source Orphanet at www.orpha.net). ${ }^{21}$ A registry of sequence variants was developed online at http://fmf.igh.cnrs.fr/ISSAID/infevers to assist the molecular geneticist. It provides a comprehensive and updated list of gene variants and a reference database for the mutation nomenclature, but an accurate phenotype-genotype correlation is not available. In addition, clinical reference centres were formally nominated or are recognised in several countries (France, Italy, Spain, UK, Germany, Turkey, Israel, USA...). As HRFs are rare diseases mostly caused by single-nucleotide substitutions, genetic testing should be referred to specialised laboratories to ensure that pertinent tests are performed and proper information is reported to clinicians, particularly those inexperienced with HRFs. These laboratories should work within a comprehensive quality management system (accreditation), use validated methods, participate annually in interlaboratory comparisons such as external quality assessment and proficiency testing for HRF and/ or the relevant techniques (eg, DNA sequencing) and define a typical turn-around time.

\section{Testing strategy}

Most laboratories focus the molecular analysis on mutational hot-spot regions in various genes. Recommendations on the reference sequence to be used for analysis and for the extent of the initial mutation screening are provided in table 1 . The minimum diagnostic screen should include variants that are clearly shown to be pathogenic and that are frequently identified in patients. Although this screening recommendation is valid worldwide, the ethnic background of the patient needs to be considered. For instance, the four clearly pathogenic $M E F V$ variants are almost exclusively found in Mediterranean populations, while the frequency of the debated p.Glu148Gln (NM_000243.2:c.442G $\rightarrow$ C) variant is as high as $20 \%$ in Asiatic countries. ${ }^{22}$ The p.Phe479Leu (NM_000243.2:c. 1437C $\rightarrow$ G) is especially relevant in Greek and Iranian patients. $^{22}$ The p.Pro75Leu (NM_001065.3:c.224C $\rightarrow \mathrm{T}$ ) (usual name P46L) of TNFRSF1A is commonly found in Arabic and African populations. ${ }^{23}$ Accordingly, many laboratories have adopted a two-step strategy-that is, an initial search for the most common pathogenic variants followed, if necessary, by an extended search spanning the complete coding sequence of the various genes. For the $M E F V$ gene, there is limited utility in searching for rare variants for patients with clinically established FMF and no mutations in exons 2, 3, 5 and 10.

\section{Techniques}

A variety of techniques are used to identify HRFs gene sequence variations but direct mutation analysis by DNA sequencing is the method employed by most laboratories. Other methods include PCR with restriction enzyme digest, allele-specific PCR, PCRsingle-strand conformation polymorphism and primer extension and reverse hybridisation-based kits. Commercially available kits should specify whether they are CE-IVD (in vitro diagnostics) marked or Food and Drug Administration approved. General guidelines ${ }^{18}$ should be followed. No standard primer set is recommended for amplification of the essential regions in HRF genes, but:

1. Primer sequences should be regularly checked for underlying single-nucleotide polymorphisms (SNPs) particularly for highly polymorphic exons of the HRF genes (eg, $M E F V$ exons 2 or 5$)$.

2. PCR primer design should avoid possible amplification of sequences from homologous genes (eg, NLRP genes).

\section{INTERPRETATION}

\section{Classification and validation of HRF sequence variants}

The Human Genome Variation Society (HGVS) has recommended avoiding the use of 'mutation' and polymorphism' as 


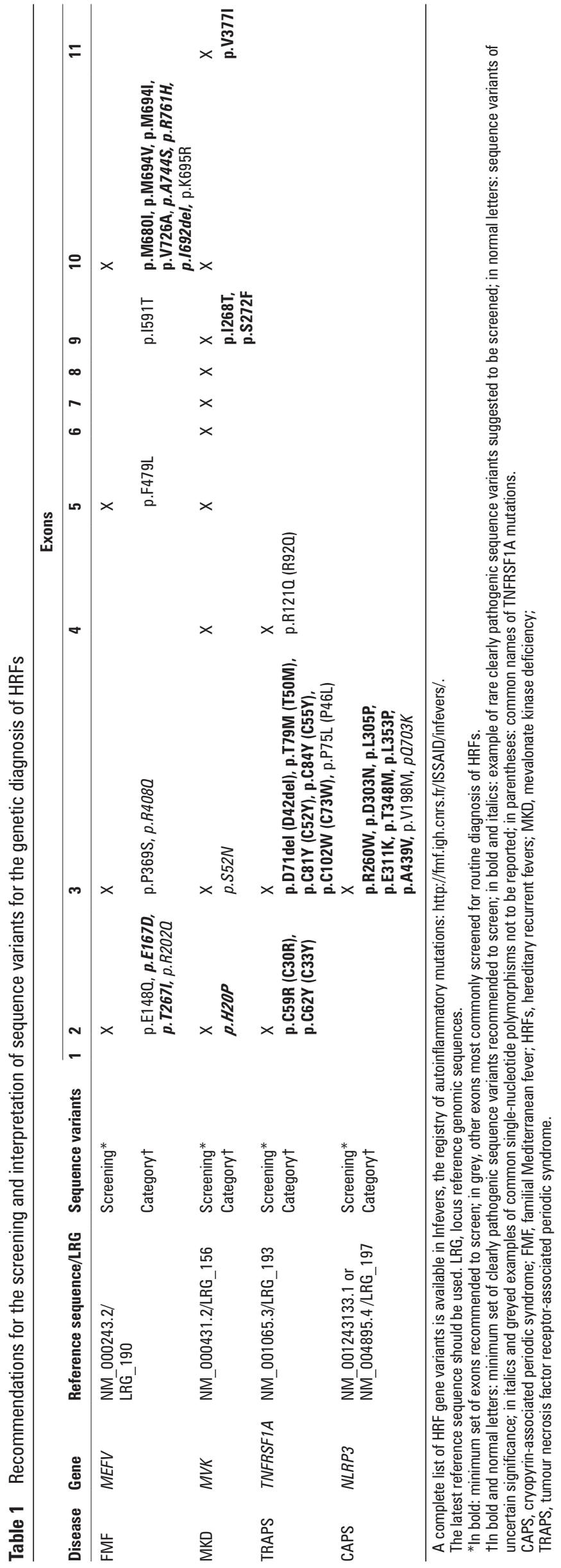

terms with opposite meanings (pathogenic vs non-pathogenic), since functional studies are scarce or unavailable for most of the sequence variants to evaluate their pathogenicity. HRF sequence variants are associated with a broad range of phenotypes, but only a small proportion of them have been clearly shown to be the direct cause of the disease. The most common variants seen in the HRF genes are non-synonymous nucleotide changes and with the exception of $\mathrm{MKD}$, no large structural mutations (deletions, duplications, rearrangement) have been reported. ${ }^{24} 25$ This is probably because such deleterious pathogenic variants would not be tolerated in genes that regulate host defence pathways.

We have established a classification of gene variants based on the expertise of HRF diagnostic laboratories and on the review of current publications (table 1). Interpretation should differentiate the following:

1. Clearly pathogenic variants. Genetic confirmation of HRFs is more straightforward in cases of sequence variants that associate with a well-recognised HRF phenotype (eg, p.Met694Val, NM_000243.2:c.2080A $\rightarrow$ G in MEFV), or those clearly altering the protein structure (eg, cysteine mutations in TNFRSF1A; deletions, insertions in MVK). When evidence is sufficient, laboratories should clearly indicate when a detected variant (or combination of variants) is predicted to cause HRFs. If the variant has been associated with severe or moderate symptoms, references could be provided but with the note that this does not necessarily predict the individual's disease phenotype.

2. Variants of uncertain significance. These include debated frequent variants and rare or private variants.

a. Variants that have been initially published as pathogenic but later reassigned. It is not unusual that some variants that have been initially described as diseaseassociated are now found to be common in the general population, or do not segregate with the phenotype in multiplex families, or do not have much effect on the normal function of the protein. Well-known examples are p.Glu148Gln (NM_000243.2:c.442G $\rightarrow$ C) of $M E F V$, p.Arg121Gln (NM_001065.3:c.362G $\rightarrow$ A) or p.Pro75Leu (NM_001065.3:c.224C $\rightarrow \mathrm{T}$ ) (usual names R92O and P46L, respectively) of TNFRSF1A and p.Val198Met (NM_001243133.1:c.592G $\rightarrow \mathrm{A}$ ), of NLRP3 (also known as V200M).

b. Variants with no reliable information or new variants. Testing unaffected parental samples for the presence of a new variant is fairly straightforward and a very informative way to assess their contribution to HRF. The frequency of rare variants in ethnically matched populations could be evaluated in silico by searching various databases such as dbSNP (http://www.ncbi. nlm.nih.gov/projects/SNP/), 1000 Genomes Pilot project and PubMed. There are also other web-based tools for evaluating the degree of evolutionary conservation (eg, Genomics Evolutionary Rate Profiling: GERP, http:// mendel.stanford.edu/sidowlab/downloads/gerp/index. $\mathrm{html}$ ) and the functional impact on the protein (eg, PolyPhen-2 http://genetics.bwh.harvard.edu/pph2/). For variants that are suspected to affect transcript splicing the patient's cDNA should be analysed if available.

3. Variants that are clearly not the genetic cause should not be reported. Coding region SNPs frequent in the general population are often encountered. Examples are p.Arg202Gln (NM_000243.2: c.605G $\rightarrow$ A, minor allele frequency (MAF)=0.18) of $M E F V$ which is in linkage disequilibrium with p.Met694Val, NM_000243.2:c.2080A $\rightarrow \mathrm{G}^{26}$ 
LABORATORY identifiers and contact information

GENETIC DIAGNOSIS OF [ACRONYME HRF]

(Extended name HRF)

Name of referring health care professional :

Date of receipt of the sample :

Subject: [Family name, given name patient]

Date of birth: ----------

Patient ID: [internal ID]

Type of sample: [DNA/blood $]$

Indication for testing:

Method: Method (sequencing, kit etc...) and extent of the screening (exons ---) of the [HRF] gene (ref seq: NM_XXXX.X). This allows identification of XX\% of the known mutations.

Results: Two [type of the variants] were found

Mutations and genotype to be given using HGVS nomenclature

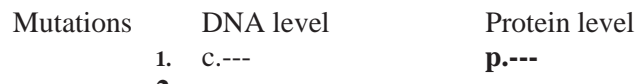

2. c.--- p.---

Genotype:

$$
\begin{array}{ll}
\text { p.[---];[---] } & \text { if phased not allelic } \\
\text { p.[---;---] } & \text { if phased allelic } \\
\text { p.[---(;)---] } & \text { if not phased }
\end{array}
$$

\section{Interpretation:}

[To be reported here as suggested in table 2]

Recommendations: Genetic counseling is advised.

Date of report

Name of the molecular geneticist(s)

Figure 1 A proposed model for reporting the genetic diagnosis of hereditary recurrent fevers (HRFs). This is an example of a recessive disease, showing the minimal items to be reported. A report should ideally highlight the major findings and not exceed one page. HGVS, Human Genome Variation Society.

p.Ser52Asn (NM_000431.1:c.155G $\rightarrow$ A, MAF=0.09) of MVK and p.Gln703Lys (NM_001243133.1:c.2107C $>\rightarrow$ A, $\mathrm{MAF}=0.02)$ of NLRP3. ${ }^{27}$ We believe that reporting these SNPs in the context of genetic diagnosis of HRF might mislead the report recipient.

\section{Genetic confirmation of the clinical diagnosis}

As for any Mendelian conditions, the definitive genetic diagnosis of HRFs is based on the finding of unambiguous mutations in the causative genes. Theoretically, identification of one single pathogenic variant in dominant diseases, homozygosity or compound heterozygosity (confirmed by studying the parental alleles) in recessive disorders, should be enough to confirm the diagnosis. Of note, finding a single $M E F V$ pathogenic variant in patients of Mediterranean origin does not exclude the possibility of disease-causing mutations in other HRF genes. ${ }^{28}$ However, interpretation of a result should always take into account the sensitivity of the molecular screening strategy. Failure to identify a causal mutation in a given gene can almost never exclude the diagnosis. Indeed, the entire gene is not sequenced in most routine settings and even complete gene sequencing based on PCR technology could miss a pathogenic variant (primer variants, inversion of exons, duplication of exons...) Likewise mosaicism will be most likely missed by standard sequencing. 
Table 2 Guidelines for reporting and interpreting genetic results in the four main HRFs

\begin{tabular}{|c|c|}
\hline a & Report of results \\
\hline \multicolumn{2}{|l|}{ Patients with symptoms } \\
\hline \multicolumn{2}{|l|}{ Recessive diseases (FMF or MKD) } \\
\hline \multicolumn{2}{|l|}{ Two clearly pathogenic variants } \\
\hline Phased & $\begin{array}{l}\text { The patient is (homozygote or compound heterozygote) for two } \\
\text { clearly pathogenic variants in the (MEFV/MVK) gene }\end{array}$ \\
\hline Not phased & $\begin{array}{l}\text { Two clearly pathogenic variants were identified in the (MEFV/MVK) } \\
\text { gene. They have (never/already) been reported in cis (complex } \\
\text { allele) }\end{array}$ \\
\hline \multicolumn{2}{|l|}{$\begin{array}{l}\text { One clearly pathogenic and } \\
\text { one VUS }\end{array}$} \\
\hline Phased & $\begin{array}{l}\text { The patient is compound heterozygote for one clearly pathogenic } \\
\text { variant and one variant of uncertain clinical significance in the } \\
\text { (MEFV/MVK) gene }\end{array}$ \\
\hline Not phased & $\begin{array}{l}\text { One clearly pathogenic mutation and one variant of uncertain clinica } \\
\text { significance were identified in the (MEFV/MVK) gene. They have } \\
\text { (never/already) been reported in cis (complex allele) }\end{array}$ \\
\hline \multicolumn{2}{|l|}{ Two VUS } \\
\hline Phased & $\begin{array}{l}\text { The patient is (homozygote or compound heterozygote) for two } \\
\text { variants of uncertain clinical significance in the (MEFV/MVK) } \\
\text { gene }\end{array}$ \\
\hline Not phased & $\begin{array}{l}\text { Two variants of uncertain clinical significance were identified in the } \\
(M E F V / M V K) \text { gene }\end{array}$ \\
\hline One clearly pathogenic variant & $\begin{array}{l}\text { One clearly pathogenic variant was identified in the (MEFV/MVK) } \\
\text { gene }\end{array}$ \\
\hline One VUS or no variant & $\begin{array}{l}\text { No pathogenic or one variant of uncertain clinical significance was } \\
\text { identified in the (MEFV/MVK) gene }\end{array}$ \\
\hline \multicolumn{2}{|l|}{$\begin{array}{l}\text { Dominant diseases (TRAPS or } \\
\text { CAPS) }\end{array}$} \\
\hline One clearly pathogenic variant & $\begin{array}{l}\text { One clearly pathogenic variant was identified in the (TNFR1SF1A/ } \\
\text { NLRP3) gene }\end{array}$ \\
\hline One VUS & $\begin{array}{l}\text { One variant of uncertain clinical significance was identified in the } \\
\text { (TNFR1SF1A/NLRP3) gene. }\end{array}$ \\
\hline
\end{tabular}

No variant

No pathogenic variant was identified in the (TNFRSF1A/NLRP3)gene

\section{Asymptomatic individuals}

Genotype consistent with HRF Adapt from above

Recessive diseases (FMF or MKD)

One sequence variant The individual is a carrier for a (clearly pathogenic variant/variant of uncertain significance) in the (MEFV/MVK) gene
Interpretation

This genotype confirms clinical diagnosis of (FMF/MKD). If relevant add: and is generally associated with a (mild or severe) phenotype

This genetic result is consistent with clinical diagnosis of (FMF/ MKD). Parental testing should resolve the issue of complex allele. If relevant add: proven homozygosity or compound heterozygosity with these two variants is generally associated with a (mild or severe) phenotype

This genotype could be consistent with clinical diagnosis of (FMF/MKD). If relevant add: and is generally associated with a (mild or severe) phenotype

This genetic result could be consistent with clinical diagnosis of (FMF/ MKD). Parental testing should resolve the issue of complex allele. If relevant add: proven homozygosity or compound heterozygosity with these two variants is generally associated with a (mild or severe) phenotype

Diagnosis relies on clinical judgement or criteria. If relevant add: possible association with a mild phenotype

Diagnosis relies on clinical judgement or criteria. Parental testing should resolve the issue of complex allele. If relevant add: possible association with a mild phenotype

Rare undetected variants may exist. Diagnosis relies on clinical judgement or criteria

Rare undetected variants may exist. Diagnosis relies on clinical judgement or criteria. Refer to an expert clinician to consider other HRFs

This genotype confirms clinical diagnosis of (TRAPS/CAPS). If relevant add: and is generally associated with a (mild/severe) phenotype

Diagnosis relies on clinical judgement or criteria. Refer to an expert clinician to consider other HRFs. If rare or new add: (parental testing/ familial segregation) may help understanding the clinical significance of this variant

Rare undetected variants may exist. Diagnosis relies on clinical judgement or criteria. Refer to an expert clinician to consider other HRFs

The individual is at risk of developing symptoms of HRF. If relevant add: or inaugural renal amyloidosis. It is recommended that acute phase reactants (CRP, SAA) and the kidney function (urine analysis) be regularly monitored

This individual is not likely to develop (MKD/FMF)

\section{REPORTING}

We recommend that genetic test results should be sent to the doctor(s) designated by the patient and not directly to the patient. The referring doctor should be invited to contact the laboratory if they have not fully understood the clinical significance of the test result. In the case of family studies, genetic results should not be communicated to other relatives without their consent. We recommend that a patient's information on genetic testing results should be given by a doctor skilled in the field of HRFs or by an expert geneticist, if available.

We strongly suggest that the reports include all items recommended by the OECD quality assurance in genetic testing (http://www.oecd.org/dataoecd/43/6/38839788.pdf) and match the international standard ISO-15189. The laboratory report should be limited to a single page, with the genetic results and interpretation highlighted and the rest in smaller characters or presented in footnotes. A model report is proposed (figure 1).

\section{Genetic results}

1. Variants should be described at both the protein and nucleotide level and should comply with the latest version of HGVS nomenclature (http://www.hgvs.org/mutnomen/). It is preferable to use the three-letter amino acid code.

2. Wherever the sequence variant name has changed owing to renumbering of the start codon, both the standard and HGVS nomenclature should be reported, to allow the referring clinician access to the relevant literature. Examples are p.Arg121Gln (NM_001065.3:c.362G $\rightarrow$ A) (common name R92O) of TNFRSF1A and p.Val198Met 
(NM_001243133.1:c.592G $\rightarrow$ A) (also known as V200M) of NLRP3.

3. The number for the reference sequence used should be provided.

4. A brief description of the testing technique and its clinical and analytical sensitivities for the specific population being screened (ie, extent of mutation screening and coverage of disease-associated mutations, region and limits of detection) should be provided.

\section{Interpretative comment}

Typical cases together with suggested clinical interpretation are listed in table 2. Reports should state, at a minimum, if the genotype is consistent or not with a diagnosis of HRF. Where the diagnosis is not confirmed genetically, the report should not state that a diagnosis of HRF is excluded. In this situation diagnosis relies on clinical judgement or criteria.

Additional comments in the report may refer to the presumed effect of the sequence variant on protein function and suggest further genetic testing or clinical management. Comments related to genotype-phenotype correlations are appropriate if there is enough evidence in the literature to support them. They are critical when discussing the risk of amyloidosis in patients with FMF with mutations affecting codons 680 or 694 of the $M E F V$ gene, in patients with TRAPS with cysteine pathogenic variants and in familial cases of HRF with a history of amyloidosis. A comment on mild disease outcome can be considered for genotypes with mild or low penetrance sequence variants.

It is preferable to recommend referral to genetic counselling and/or to a clinical reference centre rather than to comment directly on treatment options or the predicted risk for the offspring or other family members. The clinical reference centres are better placed to fully discuss these pertinent issues. Testing should be offered to other symptomatic family members and to the parents where this can help with interpretation of the proband's results.

\section{Clerical information}

The other important items suggested by the OECD are summarised below:

- More than one identifier that unequivocally links the report to the patient (name, date of birth, internal reference laboratory number).

- The name of the referring healthcare professional and contact information.

- The indication for testing and specific medical information where it is relevant.

- The date of receipt of the sample and of report issuing.

- The laboratory contact information and the identity of the individual approving the report.

\section{CONCLUSION}

A consensus set of best practice guidelines has been developed for molecular genetic testing of HRFs based on feedback received from experts in this field. The guidelines described here are aimed at improving the quality of HRF molecular diagnostics and promoting harmonisation and standardisation of laboratory test reports. Understanding the molecular pathology of these diseases, their heterogeneity and genotype-phenotype correlations is steadily evolving as more data become available from large population cohorts of patients and healthy controls. A particular challenge for the genetic diagnosis of HRF will be in the interpretation of clinical relevance of variants that are found at low, but $>1 \%$, frequency in various populations. These may function as susceptibility alleles to inflammation rather than disease-associated mutations and as such may give rise to an inflammatory phenotype when inherited through digenic inheritance (in the form of double heterozygous). In that context we feel that these guidelines may need to be regularly updated.

\section{Useful links}

Infevers: Registry of autoinflammatory mutations: http://fmf. igh.cnrs.fr/ISSAID/infevers/

ISSAID: Website of the International Society of Systemic Autoinflammatory Diseases: http://fmf.igh.cnrs.fr/ISSAID/

Eurofever: Registry of autoinflammatory patients: http://www. printo.it/eurofever/

Orphanet: Reference portal for information on rare diseases and orphan drugs: http://www.orpha.net/

HGVS: Reference for the nomenclature for the description of sequence variants: http://www.hgvs.org/mutnomen/

EMON: European Molecular Genetics Quality Network: http:// www.emqn.org/emqn/

For FMF: The Centre of Arab Genomic Studies (http://www. cags.org.ae) and the Israeli National Genetic Database (http:// www.goldenhelix.org/server/israeli/)

Contributors IT led the project, organised the meeting, wrote the first draft of the paper and was aided by YS, LO and IA. BB and HH edited the paper for English language. All authors participated in the development of the guidelines and contributed to the writing of the paper.

Acknowledgements This study was supported and performed within the framework of the EU projects EuroGentest (FP6-512148), Eurotraps (coordination theme 1 (health) of the European Community's FP7, grant agreement number HEALTH-F2-2008-200923 and la Région Languedoc-Roussillon) and Eurofever (EAHC, Project No2007332). It was also supported by the French Ministry of Health. The authors are indebted to Drs Catherine Dodé, Laurence Cuisset and Isabelle Jéru from the GenMAI network for the elaboration of the clinical questionnaire for the genetic diagnosis of HRFs. The authors thank Dr Robert Elles for reviewing the paper and Outi Kamarainen and Simon Patton for overseeing the HRF EQA scheme of EMON and for the logistic support of the meeting.

Collaborators Hatem El Shanti, Marianne Jakobsen, Kostas Kostantopoulos, Davide Martorana, Maria Grazia Pomponi, Alison Bybee, Catherine Rydlewski

\section{Funding EU.}

Note added in proof While this manuscript was in press, a new article by Verma et al demonstrated a possible functional role of p0703K (0705K) in the regulation of inflammation. This variant should therefore be considered a VUS (should be in normal letters in table 1).The 0705K Polymorphism in NLRP3 Is a Gain-of-Function Alteration Leading to Excessive Interleukin-1 $\beta$ and IL-18 Production.Verma D, Särndahl E, Andersson H, et al.PLoS One. 2012;7:e34977. Epub 2012 Apr 17.

Provenance and peer review Not commissioned; externally peer reviewed.

Correction notice This article has been corrected since it was published Online First.

\section{Author affiliations}

${ }^{1}$ Heller Institute of Medical Research, Sheba Medical Center, Tel Hashomer, Israel

${ }^{2}$ Amyloid Center, Biotechnology Research Laboratories, Fondazione IRCCS, Policlinico

San Matteo, Pavia, Italy

${ }^{3}$ National Human Genome Research Institute, Bethesda, Maryland, USA

${ }^{4}$ Department of Molecular Genetics, The Children's Hospital at Westmead, Sydney, Australia

${ }^{5}$ Department of Human Genetics, LADR-MVZ Recklinghausen, Germany ${ }^{6}$ Laboratorio di Genetica Molecolare, Istituto Giannina Gaslini, Genova, Italy ${ }^{7}$ Department of Molecular Genetics, Laboratoire Cerba, Cergy-Pontoise, France ${ }^{8}$ Department of Human Genetics ULB, Hopital Erasme, Brussels, Belgium

${ }^{9}$ UO Pediatria II - Reumatologia, Istituto G Gaslini, Genova, Italy

${ }^{10}$ Department of Pediatrics, Polish-American Children's Hospital Jagiellonian University Medical College, Poland

${ }^{11}$ Department of Paediatric and Paediatric Rheumatology, Reference center for autoinflammatory diseases CEREMAI, Kremlin-Bicêtre Hospital, APHP, University of Paris SUD, Paris, France

12Pronto diagnostics, Tel Aviv, Israel 
${ }^{13}$ Heller Institute of Medical Research, Sheba Medical Center, Tel-Hashomer, Tel-Aviv University, Tel-Aviv, Israel

${ }^{14}$ Molecular Diagnostic Laboratory, Service of Medical Genetics, Geneva University Hospitals

${ }^{15}$ Department of Pediatrics, Kyoto University Graduate School of Medicine, Kyoto, Japan

${ }^{16}$ Department of Pediatric Rheumatology and Nephrology at Hacettepe University, Ankara, Turkey

${ }^{17}$ Department of Molecular Genetics, Function and Therapy, Cyprus Institute of Neurology and Genetics, Nicosia, Cyprus

${ }^{18}$ Department of Clinical Genetics, Copenhagen University Hospital, Copenhagen, Denmark

${ }^{19}$ National Amyloidosis Centre, UCL Medical School, London, UK

${ }^{20}$ Center of Medical Genetics and Primary Health Care, Yerevan, Armenia

${ }^{21}$ Genome Diagnostics, Biomedical Genetics, University Medical Center Utrecht, Utrecht, The Netherlands

${ }^{22}$ Medical University Innsbruck, Sektion Humangenetik, Innsbruck, Austria

${ }^{23}$ Molecular Diagnostic Laboratory, Service of Medical Genetics, Geneva University Hospitals, Genève, Switzerland

${ }^{24}$ Department of Pediatrics, University of California at San Diego, La Jolla, California, USA

${ }^{25}$ CHRU Montpellier, INSERM U844, Université UM1, Montpellier, France

\section{REFERENCES}

1. Fietta P. Autoinflammatory diseases: the hereditary periodic fever syndromes. Acta Biomed 2004;75:92-9.

2. Simon A, van der Meer JW. Pathogenesis of familial periodic fever syndromes or hereditary autoinflammatory syndromes. Am J Physiol Regul Integr Comp Physiol 2007;292:R86-98.

3. Ben-Chetrit E, Levy M. Familial Mediterranean fever. Lancet 1998;351:659-64.

4. Sarrauste de Menthiere C, Terriere S, Pugnere D, et al. INFEVERS: the Registry for FMF and hereditary inflammatory disorders mutations. Nucleic Acids Res 2003;31:282-5

5. Touitou I, Lesage S, McDermott M, et al. Infevers: an evolving mutation database for auto-inflammatory syndromes. Hum Mutat 2004;24:194-8.

6. Milhavet $\mathbf{F}$, Cuisset L, Hoffman HM, et al. The infevers autoinflammatory mutation online registry: update with new genes and functions. Hum Mutat 2008;29:803-8.

7. Koné-Paut I, Hentgen V, Guillaume-Czitrom S, et al. The clinical spectrum of 94 patients carrying a single mutated MEFV allele. Rheumatology (Oxford) 2009;48:840-2.

8. Booty MG, Chae JJ, Masters SL, et al. Familial Mediterranean fever with a single MEFV mutation: where is the second hit? Arthritis Rheum 2009;60:1851-61.

9. Marek-Yagel D, Berkun Y, Padeh S, et al. Clinical disease among patients heterozygous for familial Mediterranean fever. Arthritis Rheum 2009;60:1862-6.

10. Grandemange S, Soler S, Touitou I. Expression of the familial Mediterranean fever gene is regulated by nonsense-mediated decay. Hum Mol Genet 2009;18:4746-55.

11. Medlej-Hashim M, Nehme N, Chouery E, et al. 1 Novel MEFV transcripts in Familial Mediterranean fever patients and controls. BMC Med Genet 2010;11:87.
12. Grateau G, Pêcheux $C$, Cazeneuve $C$, et al. Clinical versus genetic diagnosis of familial Mediterranean fever. QJM 2000;93:223-9.

13. Federici L, Rittore-Domingo C, Koné-Paut I, et al. A decision tree for genetic diagnosis of hereditary periodic fever in unselected patients. Ann Rheum Dis 2006:65:1427-32.

14. Touitou I, Rittore C, Philibert L, et al. An international external quality assessment for molecular diagnosis of hereditary recurrent fevers: a 3-year scheme demonstrates the need for improvement. Eur J Hum Genet 2009;17:890-6.

15. Field MJ, Lohr KN. Clinical Practice Guidelines. Directions for a New Program, Institute of Medicine, Washington, DC: National Academy Press 1990:38.

16. King C, Barton DE. Best practice guidelines for the molecular genetic diagnosis of Type 1 (HFE-related) hereditary haemochromatosis. BMC Med Genet 2006; 7:81.

17. Dequeker $\mathbf{E}$, Stuhrmann M, Morris MA, et al. Best practice guidelines for molecular genetic diagnosis of cystic fibrosis and CFTR-related disorders-updated European recommendations. Eur J Hum Genet 2009;17:51-65.

18. Dutt T, Burns S, Mackett N, et al. Application of UKHCDO 2004 guidelines in type 1 von Willebrand Disease-a single centre paediatric experience of the implications of altered or removed diagnosis. Haemophilia 2011;17:522-6.

19. Samuels J, Ozen S. Familial Mediterranean fever and the other autoinflammatory syndromes: evaluation of the patient with recurrent fever. Curr Opin Rheumatol 2006;18:108-17.

20. Gattorno M, Sormani MP, D'Osualdo A, et al. A diagnostic score for molecular analysis of hereditary autoinflammatory syndromes with periodic fever in children. Arthritis Rheum 2008;58:1823-32.

21. Weinreich SS, Mangon R, Sikkens JJ, et al. (Orphanet: a European database for rare diseases). Ned Tijdschr Geneeskd 2008;152:518-19.

22. Touitou I. The spectrum of Familial Mediterranean Fever (FMF) mutations. Eur J Hum Genet 2001:9:473-83.

23. Tchernitchko D, Chiminqgi M, Galactéros $F$, et al. Unexpected high frequency of P46L TNFRSF1A allele in sub-Saharan West African populations. Eur J Hum Genet 2005:13:513-15.

24. Pallares-Ruiz N, Philibert L, Dumont B, et al. Combined mutation and rearrangement screening by quantitative PCR high-resolution melting: is it relevant for hereditary recurrent Fever genes? PLOS ONE 2010;5:e14096.

25. van Gijn ME, Soler $\mathrm{S}$, de la Chapelle $\mathrm{C}$, et al. Search for copy number alterations in the MEFV gene using multiplex ligation probe amplification, experience from three diagnostic centres. Eur J Hum Genet 2008;16:1404-6.

26. Aksentijevich I, Torosyan Y, Samuels J, et al. Mutation and haplotype studies of familial Mediterranean fever reveal new ancestral relationships and evidence for a high carrier frequency with reduced penetrance in the Ashkenazi Jewish population. Am J Hum Genet 1999;64:949-62.

27. Aksentijevich I, D Putnam C, Remmers EF, et al. The clinical continuum of cryopyrinopathies: novel CIAS1 mutations in North American patients and a new cryopyrin model. Arthritis Rheum 2007:56:1273-85.

28. Touitou I, Hentgen V, Koné-Paut I. Web resources for rare auto-inflammatory diseases: towards a common patient registry. Rheumatology (Oxford) 2009; 48:665-9. 